\title{
Controlling the Interference of Multiple Molecular Orbitals in High-Harmonic Generation
}

\author{
H. J. Wörner, J. B. Bertrand, P. Hockett, P. B. Corkum, and D. M. Villeneuve \\ Joint Laboratory for Attosecond Science, National Research Council of Canada and University of Ottawa, \\ 100 Sussex Drive, Ottawa, Ontario, Canada KlA OR6
}

(Received 2 November 2009; revised manuscript received 9 March 2010; published 11 June 2010)

\begin{abstract}
We demonstrate a new method to investigate the origin of spectral structures in high-harmonic generation. We report detailed measurements of high-harmonic spectra in aligned nitrogen and carbon dioxide molecules. Varying the wavelength and intensity of the generating laser field, we show that the minimum in aligned $\mathrm{N}_{2}$ molecules is nearly unaffected, whereas the minimum in aligned $\mathrm{CO}_{2}$ molecules shifts over more than $15 \mathrm{eV}$. Our quantitative analysis shows that both the interference of multiple orbitals and their structural characteristics affect the position of the minimum. Our method provides a simple approach to the investigation of the high-harmonic generation process in more complex molecules.
\end{abstract}

DOI: 10.1103/PhysRevLett.104.233904

High-harmonic generation (HHG) has become a tool to investigate the electronic and geometric structure of molecules on an ultrafast time scale [1-4]. HHG occurs when an electron is removed from the parent molecule under the action of an intense laser field and is forced to recollide with the parent less than one period of the field oscillations later [5]. The emission of radiation results from the recombination of an electron and a molecular ion and thus constitutes an inverse photoionization process [6-8]. Consequently, it is highly sensitive to the electronic structure of the target with the advantage that a broad range of photoelectron energies are probed simultaneously. Since the technique can be combined with nonadiabatic alignment, measurements can be performed in the molecular frame, revealing the detailed electronic structure of molecules [9]. An important example of structural information contained in high-harmonic spectra is the interference of the returning electron wave with two atomic centers [10]. This effect has been predicted and observed in $\mathrm{H}_{2}$ [11] but whether it is applicable to larger molecules remains an object of debate. A spectral minimum observed in $\mathrm{CO}_{2}$ molecules aligned parallel to the generating laser field was found at $39 \mathrm{eV}$ in Ref. [2], at $54 \mathrm{eV}$ in Ref. [12], and in a range of intermediate positions, depending on the intensity, in Ref. [4]. Recent theoretical work suggests ionization from multiple orbitals to be responsible for the intensity dependence of the minimum position [4], while other work suggests that the effect of the strong laser field on a single orbital can explain the intensity dependence [13].

We propose and demonstrate a new method to identify and control spectral structures observed in HHG from aligned molecules. We perform detailed measurements of the high-harmonic spectra of aligned $\mathrm{N}_{2}$ and $\mathrm{CO}_{2}$ molecules at two different wavelengths and a range of intensities. We report that the minimum in $\mathrm{N}_{2}$ is largely independent of both the fundamental wavelength and the intensity which indicates that this minimum is associated with the electronic structure of the molecule. In contrast, we show that the minimum in $\mathrm{CO}_{2}$ strongly depends on
PACS numbers: 42.65.Ky, 32.80.Wr, 33.15.Mt, 33.80.Rv

both the intensity and the wavelength and is always located in the vicinity of the cutoff. This clearly demonstrates its origin as interference between channels originating in different orbitals. Varying the wavelength of the fundamental field allows us to vary the relative phase of the highharmonic emission channels, thereby controlling the photon energy at which the destructive interference occurs. Our results show that in addition to the interference of multiple orbitals, the structure of the highest occupied molecular orbital (HOMO) affects the position of the minimum. Indeed, the interference of the recombining electron with the 2-center HOMO results in a strong phase variation of the recombination matrix element with photon energy.

The experimental setup consists of a chirped-pulse-amplified titanium-sapphire laser system, a high-energy optical parametric amplifier (HE-TOPAS, Light Conversion), and a vacuum chamber for generation and characterization of high-harmonic radiation. The laser system provides pulses at $800 \mathrm{~nm}, 12 \mathrm{~mJ}, 32 \pm 2 \mathrm{fs}, 50 \mathrm{~Hz}$. The TOPAS is pumped using $8 \mathrm{~mJ}$ and provides $1200 \mathrm{~nm}$ pulses of $40 \pm$ 5 fs duration. The remaining energy is split into two beams of adjustable intensity. The molecules are impulsively aligned using a stretched $800 \mathrm{~nm}$ pulse (duration $\sim 80 \mathrm{fs}$ ) with a peak intensity of $5 \times 10^{13} \mathrm{~W} / \mathrm{cm}^{2}$. The highharmonic spectra are recorded at a time delay corresponding to the maximal alignment at the rotational half-revival $\left(\sim 4.1 \mathrm{ps}\right.$ in $\mathrm{N}_{2}$ and $\sim 21.2 \mathrm{ps}$ in $\left.\mathrm{CO}_{2}\right)$. The degree of alignment is estimated to be $\left\langle\cos ^{2} \theta\right\rangle=0.6-0.65$. A rotatable wave plate is used to adjust the relative polarization between the alignment and HHG pulses. The pulses are focused $\sim 1 \mathrm{~mm}$ below and slightly before the orifice of a pulsed nozzle generating a supersonic expansion. This setup minimizes the effects of phase mismatch and reabsorption of the high-harmonic radiation and leads to the observation of the single-atom response [14]. The highorder harmonics are recorded using an extreme-ultraviolet spectrometer, a microchannel plate detector, and a chargecoupled device camera [9]. When converting the pixel 
scale of the camera to the energy scale, we correct the spectral intensity to preserve the integral under the spectrum.

Figure 1 shows high-harmonic spectra of nitrogen molecules aligned parallel to the polarization of the generating field (blue line) or unaligned (green line) for different fundamental wavelengths and intensities. The spectrum shown in Fig. 1(a), recorded using a fundamental wavelength of $800 \mathrm{~nm}$, reveals a weak minimum at H25 (39 \pm $2 \mathrm{eV})$ as observed previously $[1,9,15]$. Turning to 1200 nm, Fig. 1(b) shows a clear minimum for a photon energy of $38 \pm 2 \mathrm{eV}$ for both aligned and unaligned molecules. In Fig. 1(c) the minimum is shifted to $41 \pm 2 \mathrm{eV}$ for both aligned and unaligned molecules.

We find that the minimum in $\mathrm{N}_{2}$ is almost independent of wavelength and intensity, similar to the minimum in argon $[16,17]$. This shows that the minimum is associated with the electronic structure of the molecule, rather than with electronic dynamics induced by the laser field. A weak dependence on the intensity could be attributed to the effect of the strong laser field on the wave function of the recombining electron or that of the molecular cation, owing to the energetic proximity of the cationic ground $(X)$ and first excited $(A)$ states $\left(E_{A}-E_{X} \approx 1.3 \mathrm{eV}\right.$ [18]). Moreover, the minimum is most pronounced at the lowest intensity we have used [Fig. 1(b)], suggesting that it is associated with the structure of the HOMO. Also, the overall variation of the harmonic intensity, increasing

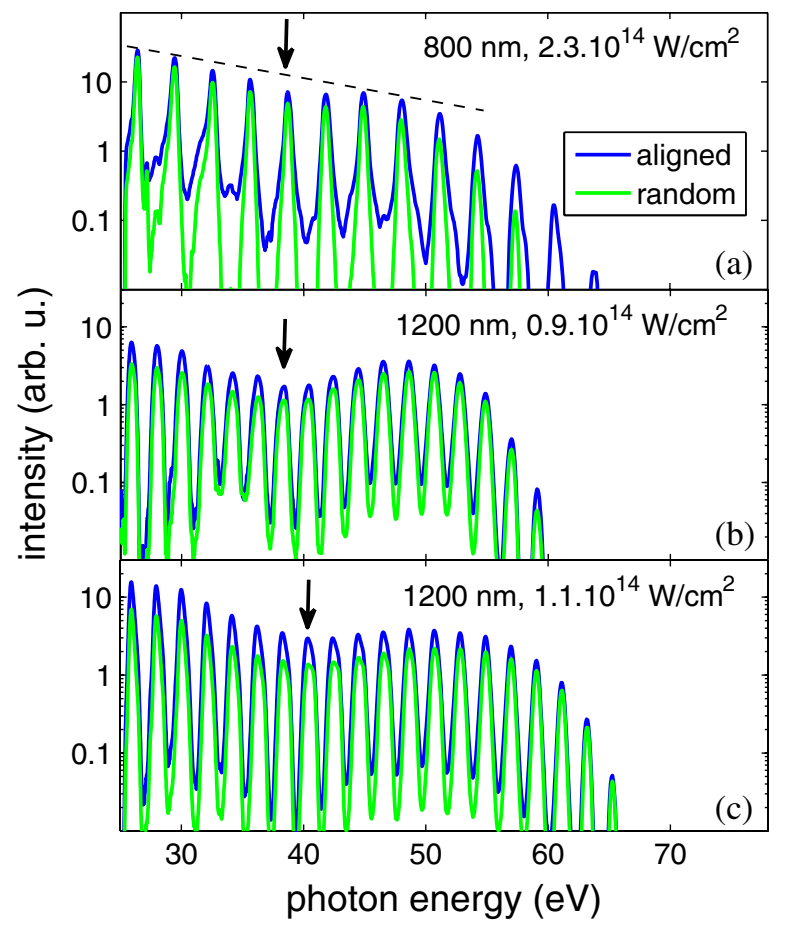

FIG. 1 (color online). High-harmonic spectra of $\mathrm{N}_{2}$ molecules aligned parallel (blue curves) or randomly aligned (green curves) to the polarization of the generating laser field. The wavelength and intensities of the generating pulse are indicated in each figure. The arrows mark the position of the minima. monotonically from perpendicular to parallel alignment (not shown), is consistent with HOMO [9].

Figure 2(a) shows spectra of $\mathrm{CO}_{2}$ molecules aligned parallel to the generating field (blue line) or unaligned (green line). At $800 \mathrm{~nm}$ and $\sim 2.1 \times 10^{14} \mathrm{~W} / \mathrm{cm}^{2}$ [Fig. 2(a)], we find a strong minimum at $\mathrm{H} 27$ or $42 \pm$ $2 \mathrm{eV}$. Figures 2(b) and 2(c) both show clear minima in the high-harmonic spectra of molecules aligned parallel to the generating field. The minimum in Fig. 2(b) is at $51 \pm 2 \mathrm{eV}$ and the minimum in Fig. 2(c) is at $57 \pm 2 \mathrm{eV}$. Unlike the minimum in $\mathrm{N}_{2}$, the minimum in $\mathrm{CO}_{2}$ moves to higher photon energies with an increasing angle between the molecular axis and the direction of the generating field (not shown). This behavior is observed at $1200 \mathrm{~nm}$ and at $800 \mathrm{~nm}$ and is consistent with the pattern expected for 2-center interference [19]. However, the dependence of its position on intensity and wavelength clearly shows that the minimum cannot originate from the molecular structure alone.

The minimum observed in $\mathrm{CO}_{2}$ molecules aligned parallel to the driving field has been attributed to the electronic structure of the HOMO $[2,12,19]$ or to the interference of the channels associated with HOMO and HOMO-2 [4]. Here we show that both structural effects of the HOMO and the interference between HOMO and HOMO-2 are required to explain our observations. The three valence orbitals of $\mathrm{CO}_{2}$ in order of increasing binding energy are of $\pi_{g}$, $\pi_{u}$, and $\sigma_{u}$ symmetry and have ionization potentials of

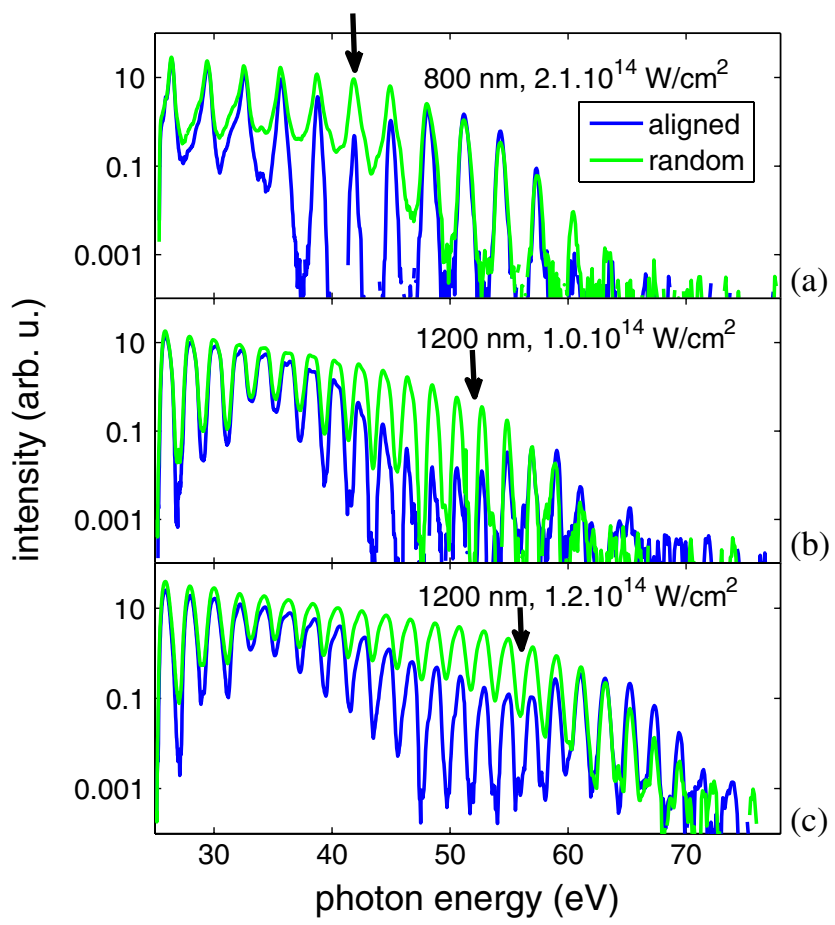

FIG. 2 (color online). High-harmonic spectra of $\mathrm{CO}_{2}$ molecules aligned parallel (blue curves) or randomly aligned (green curves) to the polarization of the generating laser field. The wavelength and intensities of the generating pulse are indicated in each figure. The arrows mark the position of the minima. 
13.8, 17.3, and $18.1 \mathrm{eV}$, respectively [18]. The angular variation of ionization rates and recombination dipoles are such that high-harmonic emission for molecules aligned at $0^{\circ}$ is dominated by HOMO and HOMO-2, while it is dominated by HOMO and HOMO- 1 at $90^{\circ}[4,20,21]$.

In order to rationalize the above observations, we have developed a simple multichannel model of high-harmonic generation $[4,20]$. Clear recent evidence for strong-field ionization from lower orbitals has demonstrated the importance of including multiple orbitals in the description of HHG $[4,22,23]$. When laser-induced transitions in the molecular ion can be neglected, the high-harmonic signal is a coherent sum of the signals generated by ionizing from and recombining to the same molecular orbital. In the spirit of the three-step model, we write the complex highharmonic amplitude as the product of three complex quantities [8,24]:

$$
d(q, \theta)=\sum_{i} a_{\mathrm{ion}, i}(q, \theta) a_{\mathrm{prop}, i}(q) a_{\mathrm{rec}, i}(q, \theta),
$$

where the sum $i$ runs over all molecular orbitals that undergo significant ionization in the laser field, $q$ is the harmonic order, and $\theta$ is the alignment angle. $a_{\text {ion, } i}(q, \theta)$ describes tunnel ionization, $a_{\mathrm{prop}, i}(q)$ the propagation, and $a_{\mathrm{rec}, i}(q, \theta)$ is the complex photorecombination matrix element in the molecular frame which characterizes the electronic structure of the molecule. We assume that the phase of $a_{\text {ion, } i}(q, \theta)$ is the same for both channels; i.e., there is no channel-specific ionization phase. This assumption will be supported by the agreement between theory and experiment. We calculate $a_{\text {prop }, i}(q)$ using the semiclassical model [25] and $a_{\mathrm{rec}, i}(q, \theta)$ using the program EPOLYSCAT [26,27].

The results of the multichannel model are illustrated in Fig. 3. Two channels are included: $\operatorname{HOMO}\left(\pi_{g}\right)$ and HOMO-2 $\left(\sigma_{u}\right)$, since Ref. [4] shows that HOMO-1 $\left(\pi_{u}\right)$ does not contribute at $0^{\circ}$. The complex molecular-frame recombination matrix element from EPOLYSCAT is averaged over a molecular alignment distribution of $\cos ^{4} \theta$ centered on the laser polarization axis. The angle dependence of the ionization probability is taken from [4]. The relative ionization probability is estimated from the ADK rates to be 0.04 . The phase of the angle-averaged recombination matrix elements is shown in Fig. 3(a). The phase of $a_{\text {rec,HOMO-2 }}$ is almost independent of the photon energy,

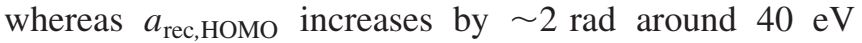
where the recombination amplitude goes through a minimum [7].

Classical trajectory calculations $[5,25]$ are used to evaluate the transit time of the electron in the continuum. As in photoionization, the high-harmonic photon energy $\Omega$ is related to the asymptotic momentum $k$ of the photoelectron by $\Omega=k^{2} / 2+I_{p}$ (in atomic units). We have calibrated the intensities in the experiment by measuring highharmonic emission from argon atoms and using the cutoff law 3.17U $U_{p}+I_{p}$ [5]. The phase difference of the electron propagation step, $\Delta I_{p} \tau$, is shown in Fig. 3(b) for the three

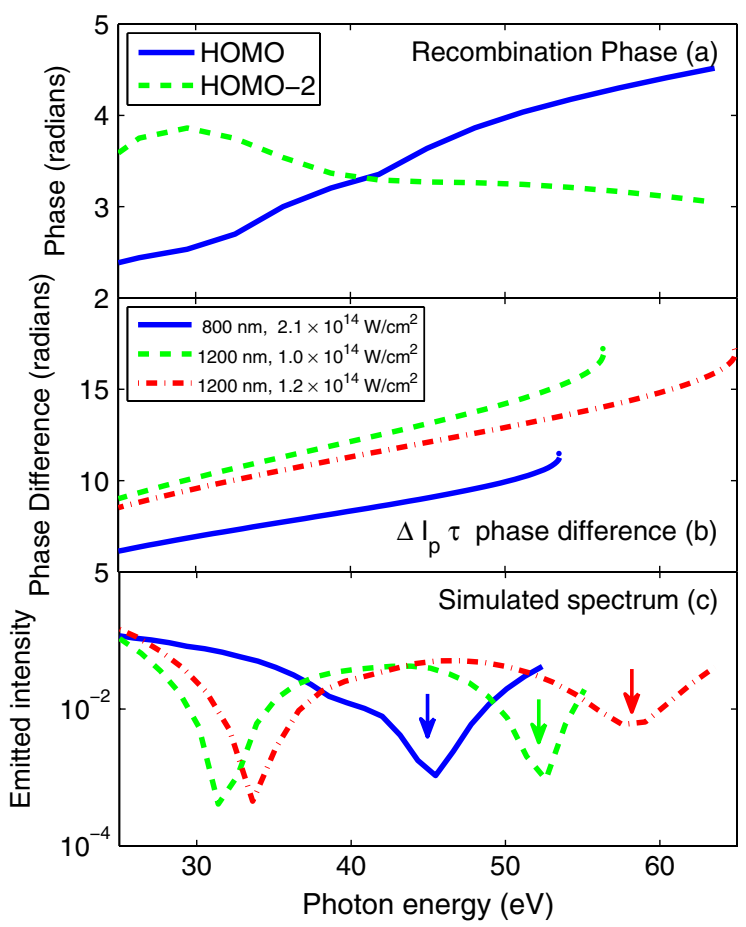

FIG. 3 (color online). Model of multichannel $\mathrm{HHG}$ from $\mathrm{CO}_{2}$ molecules aligned at $0^{\circ}$ to the laser field. (a) Phase of the angleaveraged recombination matrix elements of the two orbitals as calculated by EPOLYSCAT. (b) Phase difference acquired by the two channels during the electron excursion calculated from classical trajectories. (c) Simulated spectra from the coherent sum of the two channels. The three sets of laser parameters correspond to the experimental conditions from Fig. 2.

experimental laser conditions in Fig. 2, where $I_{p}$ is the binding energy of the involved orbitals and $\tau$ is the transit time of the electron in the continuum [28].

Figure 3(c) shows the square of the coherent sum of the electric fields from each channel up to the cutoff of HOMO. The simulated electric fields include the $\Delta I_{p} \tau$ phase and the angle-averaged complex recombination dipole moments. For each laser condition, an interference minimum is seen in the higher energy part of the spectrum (arrows). For $800 \mathrm{~nm}$, this minimum approximately corresponds to a propagation phase difference of $3 \pi$ radians because the recombination phases are almost identical. For $1200 \mathrm{~nm}$ the minima do not correspond to $5 \pi$ rad but to $\sim 4.5 \pi \mathrm{rad}$ (red dash-dotted curve) because of the difference in the recombination phases. The prediction of the $1200 \mathrm{~nm}$ spectra shows a $3 \pi$ radian minimum at lower energy; these minima are not visible in the experimental spectra because of the intensity averaging that occurs for the lower order harmonics as they are being generated over a broad range of intensities. The interference minima located close to the cutoff survive intensity averaging because the corresponding harmonic orders are only generated at the peak of the laser field. The calculated minima at 45,52 , and $58 \mathrm{eV}$ closely match the experimental minima seen in Fig. 2 at 42, 51, and $57 \mathrm{eV}$. 
The coherent sum of the multiple channels can thus reveal both structural minima due to $a_{\text {rec }, i}(q, \theta)$, or dynamical minima that occur from destructive interferences between channels. If the minimum is structural as in argon, its position is largely independent of the laser parameters including wavelength and intensity $[6,7,16,17]$. We have shown that the minimum in $\mathrm{N}_{2}$ falls in this category. If the minimum is dynamical, the interference of the orbitals can be controlled directly by intensity and wavelength through the propagation factor $a_{\text {prop }, i}(q)$. In addition to the relative phase of the two channels, the structure of the orbitals can lead to a significant phase variation of the recombination matrix element that also affects the observed minimum position.

The role of ionization phase $a_{\mathrm{ion}, i}(q, \theta)$ is not yet well understood. It is possible that there is an ionization phase that depends on the geometry and parity of the orbital involved. The ionization phase will determine the initial shape of the hole wave packet [4]. The agreement between our experiment and our model suggests that the difference in the ionization phase between the two channels in $\mathrm{CO}_{2}$ aligned parallel to the laser field is small.

In this Letter, we have shown how the variation of the fundamental wavelength allows an unambiguous identification of structural and dynamical minima. In the case of nitrogen, we have discovered that the minimum at $39 \mathrm{eV}$ primarily results from the electronic structure of the molecule. In $\mathrm{CO}_{2}$, the observed minimum results from the interference of the HOMO and HOMO-2 channels. The deviation of the minimum position from odd multiples of $\pi$ in the relative phase of the channels is attributed to a photon-energy dependent difference in the recombination phase, thereby revealing a new route to trace both structural and dynamical minima in high-harmonic spectra. When the minimum is dynamical, the position of its associated phase jump can be controlled, which is equivalent to a tunable phase mask, permitting the shaping of attosecond pulses $[19,29]$. Generally, the wavelength scaling of spectral structures in HHG spectra of aligned molecules will improve our understanding of high-harmonic generation processes in more complex molecules. In the future, a systematic variation of the wavelength could be used to investigate the role of nonadiabatic electronic dynamics [30] in high-harmonic generation. Using different wavelengths will also greatly facilitate the understanding of pump-probe spectroscopy of photochemical reactions using HHG as the probe step. The homodyne interference of the emission from the ground and the excited states [31] is controllable via the wavelength, as shown in this Letter, which will help disentangle structural and dynamical information obtained in time-resolved experiments.

We thank R.R. Lucchese, M. Y. Ivanov, and O. Smirnova for discussions. We acknowledge funding from
NSERC and AFOSR.

[1] J. Itatani et al., Nature (London) 432, 867 (2004).

[2] T. Kanai, S. Minemoto, and H. Sakai, Nature (London) 435, 470 (2005).

[3] S. Baker et al., Science 312, 424 (2006).

[4] O. Smirnova et al., Nature (London) 460, 972 (2009).

[5] P. B. Corkum, Phys. Rev. Lett. 71, 1994 (1993).

[6] T. Morishita, A.-T. Le, Z. Chen, and C. D. Lin, Phys. Rev. Lett. 100, 013903 (2008).

[7] A.-T. Le, R. R. Lucchese, S. Tonzani, T. Morishita, and C. D. Lin, Phys. Rev. A 80, 013401 (2009).

[8] M. V. Frolov et al., Phys. Rev. Lett. 102, 243901 (2009).

[9] Y. Mairesse, J. Levesque, N. Dudovich, P. B. Corkum, and D. M. Villeneuve, J. Mod. Opt. 55, 2591 (2008).

[10] M. Lein, N. Hay, R. Velotta, J. Marangos, and P. Knight, Phys. Rev. Lett. 88, 183903 (2002).

[11] S. Baker et al., Phys. Rev. Lett. 101, 053901 (2008).

[12] C. Vozzi et al., Phys. Rev. Lett. 95, 153902 (2005).

[13] P. Wei et al., Phys. Rev. A 79, 053814 (2009).

[14] A. D. Shiner et al., Phys. Rev. Lett. 103, 073902 (2009).

[15] J. Levesque, D. Zeidler, J.P. Marangos, P. B. Corkum, and D. M. Villeneuve, Phys. Rev. Lett. 98, 183903 (2007).

[16] H. J. Wörner, H. Niikura, J. B. Bertrand, P. B. Corkum, and D. M. Villeneuve, Phys. Rev. Lett. 102, 103901 (2009).

[17] S. Minemoto et al., Phys. Rev. A 78, 061402(R) (2008).

[18] K. Kimura, S. Katsumata, Y. Achiba, T. Yamazaki, and S. Iwata, Handbook of HeI Photoelectron Spectra (Japan Scientific Societies Press, Tokyo, 1981).

[19] W. Boutu et al., Nature Phys. 4, 545 (2008).

[20] O. Smirnova et al., Phys. Rev. Lett. 102, 063601 (2009).

[21] O. Smirnova, S. Patchkovskii, Y. Mairesse, N. Dudovich, and M. Y. Ivanov, Proc. Natl. Acad. Sci. U.S.A. 106, 16556 (2009).

[22] B. K. McFarland, J.P. Farrell, P. H. Bucksbaum, and M. Gühr, Science 322, 1232 (2008).

[23] A.-T. Le, R. R. Lucchese, and C. D. Lin, J. Phys. B 42, 211001 (2009).

[24] M. Y. Ivanov, T. Brabec, and N. Burnett, Phys. Rev. A 54, 742 (1996).

[25] M. Lewenstein, P. Balcou, M. Y. Ivanov, A. L'Huillier, and P. B. Corkum, Phys. Rev. A 49, 2117 (1994).

[26] F. A. Gianturco, R. R. Lucchese, and N. Sanna, J. Chem. Phys. 100, 6464 (1994).

[27] A. P. P. Natalense and R. R. Lucchese, J. Chem. Phys. 111, 5344 (1999)

[28] T. Kanai, E. J. Takahashi, Y. Nabekawa, and K. Midorikawa, Phys. Rev. Lett. 98, 153904 (2007).

[29] T. Kanai, E. J. Takahashi, Y. Nabekawa, and K. Midorikawa, Phys. Rev. A 77, 041402(R) (2008).

[30] M. Lezius et al., Phys. Rev. Lett. 86, 51 (2001).

[31] H. J. Wörner, J. B. Bertrand, D. V. Kartashov, P. B. Corkum, and D. M. Villeneuve, Nature (London) (to be published). 